\title{
Screening for hearing loss in the setting of primary care, and the most common used tests
}

\author{
Ohoud Adel Turkistani ${ }^{1 *}$, Wjdan Abduljlil Al Arqan², Rania Saad Alkhaibry', \\ Yazan Adnan Ayoub ${ }^{4}$, Rawan Mesfer Alhuthali ${ }^{5}$, Hussain Ali Alsayegh ${ }^{6}$, \\ Kholoud Khaled Momenah ${ }^{7}$, Mohammed Hamad Al Mansour ${ }^{8}$, Mohannad Sami Felemban', \\ Ahmed Ali Alqahtani ${ }^{10}$, Jasem Mohammad Ameen ${ }^{11}$
}

\begin{abstract}
${ }^{1}$ Department of Family Medicine, King Abdulaziz University Hospital, Jeddah, Saudi Arabia
${ }^{2}$ College of Medicine, Imam Abdulrahman Bin Faisal University, Dammam, Saudi Arabia

${ }^{3}$ College of Medicine, Medical University of Lodz, Lodz, Poland

${ }^{4}$ College of Medicine, Ibn Sina National College, Jeddah, Saudi Arabia

${ }^{5}$ College of Medicine, Umm Al-Qura University, Mecca, Saudi Arabia

${ }^{6}$ College of Medicine, King Faisal University, Hofuf, Saudi Arabia

${ }^{7}$ Home Healthcare Administration, Directorate of Health Affairs, Jeddah, Saudi Arabia

${ }^{8}$ College of Medicine, Najran University, Najran, Saudi Arabia

${ }^{9}$ Department of Emergency Medicine, King Faisal Hospital, Mecca, Saudi Arabia

${ }^{10}$ Department of Emergency Medicine, King Fahad General Hospital, Jeddah, Saudi Arabia

${ }^{11}$ College of Medicine, Arabian Gulf University, Manama, Bahrain
\end{abstract}

Received: 10 November 2021

Accepted: 25 November 2021

\section{*Correspondence:}

Dr. Ohoud Adel Turkistani,

E-mail: ohoudturkistani11@hotmail.com

Copyright: (C) the author(s), publisher and licensee Medip Academy. This is an open-access article distributed under the terms of the Creative Commons Attribution Non-Commercial License, which permits unrestricted non-commercial use, distribution, and reproduction in any medium, provided the original work is properly cited.

\begin{abstract}
Hearing loss is considered among the most common chronic disorders affecting people worldwide, especially older adults and geriatrics. More than half of older adults have age-related hearing loss, which worsens with age. The role of public health to estimate and manage the issue is crucial as early screening and management for hearing loss patients can be promising. The symptoms and signs of hearing loss can appear one up to two years before the significant hearing affection. Major lessons learned from this review are that elderly individuals and geriatrics are the most common targeted population for age-related hearing loss, followed by ear wax accumulation. Therefore, routine checkup for people who are 50 years for audiological disorders is a must. Associated disorders secondary to hearing loss include depression and anxiety, which significantly burden productivity over time. To our knowledge, we performed the first complex review regarding the screening for hearing loss within the setting of primary care centers and mentioned the most standard test used to diagnose and detect the issue as early as possible.
\end{abstract}

Keywords: Hearing loss, Deafness, Primary care, Audiology

\section{INTRODUCTION}

Hearing loss is considered among the most prevalent chronic diseases affecting people, especially in the elderly. ${ }^{1}$ The prevalence of hearing loss is estimated to be an average of $50 \%$ in geriatrics. The group of people older than 75 years old has a prevalence of $60 \%$ of developing sensorineural hearing loss or as known as Presbycusis. ${ }^{2}$ One of the significant issues in the current population is the increased incidence and prevalence of hearing loss compared to the past decade. Since the 1960s, the risk of developing different types of hearing loss has increased, which creates a significant burden that requires proper and dedicated investigations and early screening facilities to 
reduce the risk. ${ }^{3}$ The problems associated with hearing loss are limited to the ear, but it was also found that people who suffer from reduced hearing abilities lack good communication skills, which negatively impact their life quality. In addition to that, it was found that older adults who age-related sensorineural hearing loss developed significant levels of depression compared to healthy people in their age group. ${ }^{4}$ Therefore, audiological care is among the necessary healthcare-provided services. The role of primary health care is beyond the essential screening services and not limited to screening for diabetes mellitus or hypertension. Early screening for all body systems should be indicated to detect early manifestations of progressive diseases. ${ }^{5}$ People with specific risk factors for hearing loss should be indicated for early and continuous screening routines for their hearing abilities.

The risk factors include aging, frequent exposure to loud sounds for prolonged times, genetic factors, risk of occupational-associated hearing loss, and the usage of ototoxic drugs. ${ }^{6}$ Early signs of hearing loss are among the red lines that can be detected during primary screening. Signs include difficulty in hearing people and misunderstanding the conversations, a history of increasing the sound volume while watching TV, history of feeling stressed. In contrast, conversations include unilateral deafness, decreased ability to recognize the direction of the sound, talking loudly without recognition of sound volume, and inappropriate responses to conversations. ${ }^{1}$ The onset of early singing can be around 24 months before the significant affection of ears. Primary care physicians are the vanguard of early screening, disease detection, and primary prevention. The primary care physicians in the first instance imply the role of detecting the symptoms and signs as early as possible. Despite the current advancements in medicine, primary screening remains a need for all diseases, as early detection and management are more promising than late detection with various treatments. ${ }^{7}$ A few years ago, the preventive services task force initiated a campaign to detect auditory diseases within the community, which accepts patients through referrals from primary care centers. This project was one of the essential innovative methods for the early management of people with risk factors and hearing loss prognosis within the next few years. ${ }^{8}$ This article aims to review the literature regarding the current evidence about hearing loss screening in primary healthcare settings and highlight the most commonly used hearing tests in the screening process.

\section{LITERATURE REVIEW}

This literature review is based on an extensive literature search in Medline, Cochrane, and EMBASE databases which was performed on $27^{\text {th }}$ October 2021 using the medical subject headings (MeSH) or a combination of all possible related terms, according to the database. To avoid missing potential studies, a further manual search for papers was done through Google Scholar while the reference lists of the initially included papers. Papers discussing screening for hearing loss in the setting of primary care, and the most common used tests were screened for useful information. No limitations were posed on date, language, age of participants, or publication type.

\section{DISCUSSION}

\section{Hearing loss, etiology, and types}

Hearing loss can affect both genders at any age, from newborns to the elderly. However, there is significant dominance in geriatrics and older people who are above 60 years old. Hearing loss can develop from any part within the auditory system including, the external ear, middle ear, and inner ear. ${ }^{1}$ According to the site of the problem, the type of hearing loss is classified and identified. There are different etiologies for the various types of hearing loss. The most common presentation for hearing loss in the elderly is the sensorineural hearing loss associated with age, known as presbycusis. While in infants and newborns, the most common cause for deafness is secondary to inflammation within the middle ear, as acute otitis media or middle ear effusion. ${ }^{9}$

\section{Presbycusis and sensorineural hearing loss}

Presbycusis is hearing loss due to an affection of the inner ear system, either the cochlea or the neural auditory system. Usually, the most common risk factor leading to sensorineural hearing loss is hereditary, genetic factor's role a significant implication. However, some environmental factors and social factors may influence the process of hearing loss. ${ }^{10}$ The pathophysiology of hearing loss in Presbycusis is idiopathic to the moment. However, the current evidence supports that the overstimulation to the auditory system for long periods, especially when hereditary factors are present, is linked to the aging degeneration process. ${ }^{10}$ Presbycusis is significant in the older age group compared to young adults and children. The incidence was $66 \%$ in people over 80 years old compared to the minimal affection in adolescents. ${ }^{10}$ Although, this does not negate the presence of diseases within infants. People with Down syndrome have high liability to develop audiological manifestations, including Presbycusis, since they were born. ${ }^{11}$

\section{Conductive hearing loss}

Conductive hearing loss patients usually have a good prognosis since the problem is primarily temporary and can be managed easily compared to sensorineural hearing loss. In adults, the most common cause for conductive hearing loss is an accumulation of ear wax within the external ear or the ear canal. ${ }^{12}$ The accumulation of wax decreases the ability of good penetration from the outer environment passing to the middle ear and relying on the inner ear auditory pathway. While in children, the most typical cause for conductive hearing loss is secondary to 
infection of common colds, which by role causing collapse to the Eustachian tube and therefore affecting the middle ear; followed by a consequence of acute otitis media progressing into fluid effusion or as known as middle ear effusion. ${ }^{13}$ So, the pathophysiology behind conductive hearing loss is simple, preventable, and easily manageable, except for some cases of tumors within the middle ear or external ear, which may require surgical intervention to restore the normal function of the ear. ${ }^{14}$

\section{Mixed hearing loss}

The state of mixed hearing loss is a combination of two issues; an issue complicated by sensorineural hearing loss in addition to a conductive system problem. Although this condition is uncommon, certain medical conditions such as ear infections may complicate a mixed hearing loss prognosis. ${ }^{15}$ However, the highest prevalence and incidence for mixed hearing loss was found in people who had genetic factors for Presbycusis and developed a problem related to the external ear, such as accumulation of earwax or furunculosis within the ear canal. ${ }^{15}$ Protocols of treatment, management, and prevention are controversial but primarily symptomatic. A systemic screening for the audiological system is indicated to detect the cause of deafness, and accordingly, the treatment plan is carried out. ${ }^{15}$

\section{Tests used in hearing assessment}

\section{Pure-tone audiometry}

Pure tone audiometry, also known as pure tone audiogram, is a standard subjective test used for a long time to determine the efficacy of hearing for patients. ${ }^{16}$ The pure tone audiometry is a subjective test that depends on the patient's responses and cooperation during the examination process, and any delayed responses from the patient may impact the reliability of results. Therefore this test is better for adults rather than children. ${ }^{16}$ The idea of the test relies on testing both the air condition and bone conduction for the sound in the ear and determining the air-bone gap if present. Despite the efficacy of the pure tone audiogram, it may give unreliable results if there are some diseases present within the inner ear, such as cochlear infarction and neurological disorders within the auditory nerves. ${ }^{17}$ This test's advantages are numerous, including affordability, accessibility, ease of use, and the comprehensive reporting system. The disadvantages of pure tune audiometry are mainly due to the subjectivity of the test the dependence on the patient's response. ${ }^{18}$

\section{Bone conduction test}

The bone condition test is one of the most commonly used to determine hearing loss and hearing efficacy. The bone conduction test is conducted through the tuning fork to be applied over the bony area behind the ear, which is known as the mastoid process, or in the middle of the forehead about one up to two $\mathrm{cm}$ above the nasal ridge, and in some cases, the tuning fork may be applied over the incisor teeth to test the efficacy of bone conduction hearing. ${ }^{19}$ Also, it may be used to assess the air conduction by applying it behind the ear without touching the bone or in the midline of the face to determine the volume of sound. The tuning fork test is purely subjective depending on the patient's cooperation with the physician or the audiologist. So, this test may give good results in adults but not in children. ${ }^{20}$ The advantages of this test are that the tuning fork is portable, easy to use, affordable, can be performed in any place, and can be performed by educated non-healthcare workers. However, the significant disadvantages of the test are the subjectivity of the test, may provide inaccurate results due to the wrong technique, and cannot determine the exact degree of hearing loss within the ear. ${ }^{20}$ Therefore, the tuning fork test remains a simple test for early detection of suspected hearing loss, but it is not a confirmatory diagnostic method.

\section{Brainstem auditory evoked potential}

Brainstem auditory evoked response test is one of the most advanced facilities to objectively assess the efficacy of hearing without the need for a verbal or physical response from the patient. ${ }^{21}$ The test aims to measure the functional vitality of the auditory nerve pathway to the brainstem and the ability of sound condition to hearing center in the brain. ${ }^{21}$ The brainstem auditory evoked response depends on five relays within the neural conduction system. At first, at the level of vestibulocochlear nerve synaptic potentials, then on the level of cochlear nucleus of the vestibulocochlear nerve, then on the level of superior olivary nucleus, then on the level of lateral lemniscus axons, and finally to the relay in inferior colliculus within the brainstem. ${ }^{22}$ The significant advantage of this test is pure objectivity without a subjective response from the patient. So, it can be a good choice for children, especially newborns and infants. However, the significant disadvantages of this method are the high cost, the little availability, and the need to be present with a tertiary care hospital. So, at the level of primary care centers, this method will not be the study of choice.

\section{Screening in the setting of primary care centers}

Screening for the efficacy of hearing and detection of hearing loss in the setting of primary care centers may be challenging for various reasons. At first, primary care centers in most of the counties around the world are facilitated to serve primary and secondary medical screening tests for specific medical conditions such as hypertension, diabetes mellitus, seasonal infections, nonmajor respiratory disorders, non-major gastrointestinal disorders, and some obstetric and gynecological conditions. ${ }^{5}$ In contrast, audiological measurements may be limited and not available, so it is commonly neglected.

However, in the United States of America, it was possible to initiate a successful and sustainable campaign for hearing tests to detect early manifestations of deafness. ${ }^{23}$ 
We can learn from this exceptional experience that some facilities can be indicated within primary care centers without creating a burden in the healthcare system. At first, using easy and accessible testing methods such as the tuning fork test may be promising to detect early manifestations. Even though it may not detect the same range of issues, it remains a gold standard within the setting of non-major hospitals. ${ }^{24}$ Secondly, initiation of an effective referral system from primary care centers to tertiary care centers in case of suspected hearing loss signs or symptoms, so early management is applied before further progression. Thirdly, applying for granting funds from the government to avail more accurate hearing test devices such as pure tone audiogram within primary care centers if possible. ${ }^{25}$

The application of this impactful strategy would be promising in the early screening for audiological disorders within an early time and therefore achieving a good prognosis.

The advantages behind the accessibility, affordability, and liability of accessing hearing screening within primary centers on a routine basis to detect the associated disorders early are not only beneficial at the level of hearing. The early management of hearing disorders prevents other progressive medical conditions such as depression, anxiety, and job loss, which is a significant concern regarding the workforce, infrastructure, and economic stability within the country and governmental system. ${ }^{25}$

\section{CONCLUSIONS}

Hearing loss is considered among the most common chronic disorders affecting people worldwide, especially older adults and geriatrics. More than half of older adults have age-related hearing loss, which worsens with age. The role of public health to estimate and manage the issue is crucial and early screening and management for hearing loss patients can be promising. The routine screening protocol should start from the level of primary care centers using single and effective ways to spot diagnose the issue as early as possible.

Funding: No funding sources

Conflict of interest: None declared

Ethical approval: Not required

\section{REFERENCES}

1. Michels TC, Duffy MT, Rogers DJ. Hearing Loss in Adults: Differential Diagnosis and Treatment. Am Fam Physician. 2019;100(2):98-108.

2. Patel R, McKinnon BJ. Hearing Loss in the Elderly. Clin Geriatr Med. 2018;34(2):163-74.

3. Tucci DL, Wilson BS, O'Donoghue GM. The Growing-and Now Alarming-Burden of Hearing Loss Worldwide. Otol Neurotol. 2017;38(10):1387-8.
4. Rutherford BR, Brewster K, Golub JS, Kim AH, Roose SP. Sensation and Psychiatry: Linking AgeRelated Hearing Loss to Late-Life Depression and Cognitive Decline. Am J Psychiatry. 2018;175(3):215-24.

5. Leung MA, Flaherty A, Zhang JA, Hara J, Barber W, Burgess L. Sudden Sensorineural Hearing Loss: Primary Care Update. Hawaii J Med Public Health. 2016;75(6):172-4.

6. Lasak JM, Allen P, McVay T, Lewis D. Hearing loss: diagnosis and management. Prim Care. 2014;41(1):19-31.

7. Walling AD, Dickson GM. Hearing loss in older adults. Am Fam Physician. 2012;85(12):1150-6.

8. Chou R, Dana T, Bougatsos C, Fleming C, Beil T. Screening adults aged 50 years or older for hearing loss: a review of the evidence for the U.S. preventive services task force. Ann Intern Med. 2011;154(5):347-55.

9. Kral A. Pathophysiology of hearing loss: Classification and treatment options. Hno. 2017;65(4):290-7.

10. Kunelskaya NL, Levina YV, Garov EV. Presbyacusis. Vestn Otorinolaringol. 2019;84(4):67-71.

11. Buchanan LH. Early onset of presbyacusis in Down syndrome. Scand Audiol. 1990;19(2):103-10.

12. Sooriyamoorthy T, De Jesus O. Conductive Hearing Loss. In: StatPearls. Treasure Island (FL): StatPearls Publishing, Copyright (C) 2021, StatPearls Publishing LLC. 2021.

13. Nuttall T, Cole LK. Ear cleaning: the UK and US perspective. Vet Dermatol. 2004;15(2):127-36.

14. Cardoso FA, Monteiro EMR, Lopes LB, Avila M, Scarioli BO. Adenomatous Tumors of the Middle Ear: A Literature Review. Int Arch Otorhinolaryngol. 2017;21(3):308-12.

15. Hill-Feltham PR, Johansson ML, Hodgetts WE. Hearing outcome measures for conductive and mixed hearing loss treatment in adults: a scoping review. Int J Audiol. 2021;60(4):239-45.

16. Davies RA. Audiometry and other hearing tests. Handb Clin Neurol. 2016;137:157-76.

17. Kaga K. Auditory nerve disease and auditory neuropathy spectrum disorders. Auris Nasus Larynx. 2016;43(1):10-20.

18. Robinson DW. A review of audiometry. Phys Med Biol. 1971;16(1):1-24.

19. Bayoumy AB, de Ru JA. Sudden deafness and tuning fork tests: towards optimal utilisation. Pract Neurol. 2020;20(1):66-8.

20. Kelly EA, Li B, Adams ME. Diagnostic Accuracy of Tuning Fork Tests for Hearing Loss: A Systematic Review. Otolaryngol Head Neck Surg. 2018;159(2):220-30.

21. Polonenko MJ, Maddox RK. The Parallel Auditory Brainstem Response. Trends Hear. 2019;23:2331216519871395. 
22. Ryland MG. The amazing ear: what happens before the brainstem auditory evoked response. Am J Electroneurodiagnostic Technol. 2009;49(1):1-13.

23. Chandrasekhar SS, Tsai Do BS, Schwartz SR. Clinical Practice Guideline: Sudden Hearing Loss (Update). Otolaryngol Head Neck Surg. 2019;161(1):S1-S45.

24. Smeltzer CD. Primary care screening and evaluation of hearing loss. Nurse Pract. 1993;18(8):50-5.
25. Nieman CL, Oh ES. Hearing Loss. Ann Intern Med. 2020;173(11):Itc81-itc96.

Cite this article as: Turkistani OA, Al Arqan WA, Alkhaibry RS, Ayoub YA, Alhuthali RM, Alsayegh HA et al. Screening for hearing loss in the setting of primary care, and the most common used tests. Int $\mathbf{J}$ Community Med Public Health 2022;9:389-93. 